\title{
ANÁLISIS ESTADÍSTICO DE LA SATISFACCIÓN LABORAL EN LOS TRABAJADORES DEL ÁREA DE FARMACIA DEL HOSPITAL TEODORO MALDONADO CARBO, ECUADOR
}

\section{STATISTICAL ANALYSIS OF LABOR SATISFACTION IN WORKERS IN THE PHARMACY AREA OF THE TEODORO MALDONADO CARBO HOSPITAL, ECUADOR}

\author{
Mendoza-Mestanza Geanella Vanessa ${ }^{1 *}$ \\ ${ }^{1}$ Universidad Católica de Santiago de Guayaquil, UCSG. Guayaquil, Ecuador.
}

*Correo: gea-mendoza-923@gmail.com

\begin{abstract}
Resumen
A pesar de que la definición de calidad de vida tomó fuerza recientemente, muchos profesionales y científicos han pretendido evaluar sistemáticamente su relevancia en el comportamiento de las personas. La relevancia del estudio se fundamenta en la calidad de vida y la satisfacción laboral de los funcionarios del área de farmacia del Hospital Teodoro Maldonado Carbo. El objetivo del estudio es analizar las correlaciones entre las dimensiones de la calidad de vida y la satisfacción laboral desde la perspectiva estadística. Para el desarrollo de la investigación se emplearon los resultados propuestos en el artículo científico "Calidad de vida y satisfacción laboral de los funcionarios del área de farmacia del Hospital Teodoro Maldonado Carbo, Ecuador" (2019). Se establecieron correlaciones en función de dimensiones como la salud, felicidad, satisfacción con la vida, satisfacción económica, satisfacción con la vida familiar (con dos escalas) y satisfacción con el bienestar subjetivo. Los parámetros antes mencionados, así como las correlaciones entre variables fueron analizadas estadísticamente mediante el programa informático SPSS. Se concluye que todas las dimensiones de calidad de vida tuvieron respuestas afirmativas con respecto a esta variable, demostrando que el personal tiene una calidad de vida positiva.
\end{abstract}

Palabras clave: estadística, correlación, calidad, satisfacción laboral, farmacia.

\begin{abstract}
Although the definition of quality of life has recently taken hold, many professionals and scientists have attempted to systematically assess its relevance in people's behavior. The relevance of the study is based on the quality of life and job satisfaction of the officials in the pharmacy area of the Teodoro Maldonado Carbo Hospital. The objective of the study is to analyze the correlations between the dimensions of quality of life and job satisfaction from a statistical perspective. For the development of the research, the results proposed in the scientific article "Quality of life and job satisfaction of the officials of the pharmacy area of the Teodoro Maldonado Carbo Hospital, Ecuador" (2019) were used. Correlations were established based on dimensions such as health, happiness, life satisfaction, economic satisfaction, satisfaction with family life (with two scales) and satisfaction with subjective well-being. The aforementioned parameters, as well as the correlations between variables were statistically analyzed using the SPSS computer program. It is concluded that all the dimensions of quality of life had affirmative answers regarding this variable, demonstrating that the staff has a positive quality of life.
\end{abstract}

Keywords: statistics, correlation, quality, job satisfaction, pharmacy.

Información del manuscrito:

Fecha de recepción: 23 de marzo de 2020

Fecha de aceptación: 09 de julio de 2020

Fecha de publicación: 10 de julio de 2020 


\section{Introducción}

La calidad de vida es tan importante que su estudio nació desde hace mucho tiempo; sin embargo, esta definición tomó fuerza recientemente, muchos profesionales y científicos han pretendido evaluar sistemáticamente su relevancia en el comportamiento de las personas. Este término empezó a ser mayormente conocido en los 60's. En la actualidad este concepto tiene una gran participación dentro del sector de la salud, académico, político y en el sector empresarial. En el transcurso de los años 50's y a inicios de los 60 's los profesionales y científicos se preocuparon y enfocaron en estudiar el comportamiento humano para evaluar su bienestar, ya que el mismo estaba afectando a la industria; las ganas de querer profundizar el tema de la calidad de vida de las personas trajeron consigo a los encargados la tarea especial de diseñar indicadores estadísticos para afianzar aún más su estudio (Gómez \& Sabeh, 2018).

A inicios de los 30's se crearon dos proyectos de estudio, estos fueron direccionados a otorgarle una nueva vida a la definición de la satisfacción laboral, el primero estuvo enfocado hacia todos los colaboradores de edad adulta que vivían en un pequeño pueblo de la ciudad de Pensylvania. El investigador en ese entonces se concentró es conocer el estado de ánimo de los colaboradores de ese pueblo a través de la generación de dos preguntas ¿qué tan contentos se encuentran los colaboradores? y ¿son más felices en una ocupación que en otra?, los resultados que se rescataron de estas preguntas fueron considerados por los científicos como influyentes sobre la satisfacción laboral (Polanco, 2014).

Entre los años de 1935 y 1955 los estudios sobre la satisfacción laboral fueron más activos, cada cierto tiempo se desarrollaban más investigaciones para conocer el comportamiento de los colaboradores referente al ambiente que se producía dentro del trabajo, el hecho de que se aumentara la cantidad de estudios sobre la satisfacción de los trabajadores fue porque se consideró muy importante para el desarrollo del mercado. El pensamiento que reinaba en ese entonces era que sí el colaborador tenía la fortaleza de seguir 
manteniendo su moral, la organización tendría la posibilidad de estar libre de huelgas y generaría mayor rentabilidad.

Para medir la satisfacción laboral en tiempos pasados lo que se procedía a realizar era formular preguntas para saber cuáles eran las necesidades que consideraba más importante un trabajador dentro de su puesto de trabajo. En los años 50 se concluyó que la satisfacción laboral tenía una relación directa con el ausentismo y la rotación de trabajadores (Polanco, 2014).

La relevancia social que refleja el presente estudio es que aporta con el conocimiento acerca de la calidad de vida y la satisfacción laboral de los funcionarios del área de farmacia del Hospital Teodoro Maldonado Carbo, esto servirá para que se tomen iniciativas para motivar a los empleados y conseguir que los mismos logren tener la satisfacción suficiente dentro de su puesto de trabajo. El trabajo brindará un aporte social muy importante para que todos los funcionarios públicos $y$ privados tanto del sector de salud como del comercial puedan tener una mayor satisfacción laboral y calidad de vida.
El estudio de investigación acerca de la calidad de vida y satisfacción laboral de los funcionarios del área de farmacia tiene un impacto muy importante en la sociedad y dentro de la industria de servicios sanitarios, puesto que por medio de este trabajo se podrá obtener la información correspondiente que ayudará a mejorar la calidad de vida de los colaboradores de salud, permitiendo que ellos mismos eleven sus niveles de satisfacción conforme a las responsabilidades que tienen a su cargo. A su vez, la implicación de cambios organizacionales ayudará a mejorar la calidad de los servicios brindados actualmente en esta casa de salud.

\section{Metodología}

\subsection{Descripción metodológica}

Para el desarrollo de la presente investigación se emplearon los resultados propuestos en el artículo científico "Calidad de vida y satisfacción laboral de los funcionarios del área de farmacia del Hospital Teodoro Maldonado Carbo, Ecuador" de Mendoza-Mestanza (2019), cuyos datos fueron obtenidos mediante una investigación descriptiva, por motivo de que se estableció una caracterización de la 
situación actual del Hospital Teodoro Maldonado Carbo. No obstante, en el presente estudio se realizó una discusión particularmente diferente a la realizada en el manuscrito referenciado. Los resultados fueron analizados desde una perspectiva correlacional de las variables calidad de vida y satisfacción laboral en los funcionarios de la farmacia del Hospital Teodoro Maldonado Carbo de Guayaquil, Ecuador.

En lo que respecta al instrumento de investigación, la investigación referenciada aplicó encuestas basadas en un cuestionario diseñado para obtener la información necesaria para dar respuesta a las preguntas de investigación (Instituto de Hematología e Inmunología, 2018).

El cuestionario empleado corresponde a la "Encuesta de calidad de vida en relación a la satisfacción laboral y estilo de vida", dicho cuestionario está compuesto por 17 preguntas planteadas con la finalidad de determinar la relación existente entre calidad de vidasatisfacción laboral.

Se debe señalar que el cuestionario fue elaborado a través de las siguientes escalas: índice de calidad de vida relativo a la salud (Hennessy et al., 1994); escala de felicidad (Lyubomirsky \& Lepper, 1999), traducida al español por Vera \& Celis (2011); escala de satisfacción con la vida (Diener, 1985), traducida por la Universitat de Valéncia (2018); escala de satisfacción con la vida familiar (Zabriskie \& Ward, 2013); escala de bienestar económico subjetivo (Giarrizzo, 2010); escala de satisfacción con la vida (Diener et al., 1985); escala de satisfacción laboral (Warr, 1999); traducida por Pérez \& Fidalgo (1999); escala de satisfacción laboral (Spector, 1997). Todas las encuestas han sido validadas estadísticamente, habiéndose comprobado su fiabilidad a través del alfa de Cronbach.

\subsection{Correlaciones}

Se establecieron correlaciones en función de variables de la calidad de vida, es decir este parámetro fue evaluado según la salud, felicidad, satisfacción con la vida, satisfacción económica, satisfacción con la vida familiar (con dos escalas) y satisfacción con el bienestar subjetivo. Además, se evaluaron los aspectos sociodemográficos de los participantes. 
Los parámetros antes mencionados, así como las correlaciones entre variables fueron analizadas estadísticamente mediante el programa informático SPSS.

\subsection{Técnica Estadística}

La correlación de Pearson es una herramienta de la estadística creada con el objetivo de dar un análisis sobre la relación o el vínculo que existe entre dos variables de investigación. El análisis que se realiza se lo procede a desarrollar en un nivel por intervalos o también se puede proceder a efectuarlo a través del nivel de razón. En la tabla 1 se presentan los niveles de medición de las variables.

\section{Tabla 1.}

Niveles de medición de las variables

\begin{tabular}{cc}
\hline 1 & Correlación negativa perfecta \\
0.90 & Correlación negativa fuerte \\
0.75 & Correlación negativa considerable \\
0.50 & Correlación negativa media \\
0.25 & Correlación negativa débil \\
0.10 & Correlación negativa muy débil \\
0.00 & No existe correlación entre las variables \\
0.10 & Correlación positiva muy débil \\
0.25 & Correlación positiva débil \\
0.50 & Correlación positiva media \\
0.75 & Correlación positiva considerable \\
0.90 & Correlación positiva muy fuerte \\
1 & Correlación positiva perfecta \\
\hline
\end{tabular}

Fuente: Tomado de Hernández et al. (2014).
3. Resultados y discusión

3.1. Correlación entre las variables

En el análisis correlacional, se definieron diversas correlaciones para identificar de mejor manera la forma en que la satisfacción laboral tiene incidencia en la calidad de vida de los colaboradores del área de farmacia del Hospital Teodoro Maldonado Carbo. Según MendozaMestanza (2019), en la primera pregunta de dicho estudio se evalúa la calidad de vida desde el punto de vista de la salud, esta se correlacionó con las dos escalas de satisfacción laboral, brindando un valor de 0,514 para la primera escala y de 0,398 para la segunda escala (tabla 2). Esto quiere decir que la satisfacción laboral tiene una correlación positiva media y correlación positiva débil sobre la variable de calidad de vida desde el aspecto de salud. Esto puede deberse a que personas que consideran que no se encuentran bien en su salud perciben una mayor insatisfacción en su trabajo. 
Tabla 2.

Correlación calidad de vida según la salud - satisfacción laboral

\begin{tabular}{|c|c|c|c|c|}
\hline & & $\begin{array}{l}\text { Calidad de } \\
\text { vida según la } \\
\text { salud }\end{array}$ & $\begin{array}{l}\text { Satisfacción } \\
\text { escala } 1\end{array}$ & $\begin{array}{l}\text { Satisfacción } \\
\text { escala } 2\end{array}$ \\
\hline $\begin{array}{l}\text { Calidad de vida según la } \\
\text { salud }\end{array}$ & $\begin{array}{l}\text { Correlación de Pears on } \\
\text { Sig. (bilateral) } \\
N\end{array}$ & $\begin{array}{r}1 \\
75\end{array}$ & $\begin{array}{r}, 514^{\pi \times} \\
, 000 \\
75\end{array}$ & $\begin{array}{r}, 398^{\pi \times} \\
, 000 \\
75\end{array}$ \\
\hline Satisfacción escala 1 & $\begin{array}{l}\text { Correlación de Pears on } \\
\text { Sig. (bilateral) } \\
\text { N }\end{array}$ & $\begin{array}{r}, 514^{\star \pi} \\
, 000 \\
75\end{array}$ & 75 & $\begin{array}{r}, 969^{\times \pi} \\
, 000 \\
75\end{array}$ \\
\hline Satisfacción escala 2 & $\begin{array}{l}\text { Correlación de Pears on } \\
\text { Sig. (bilateral) } \\
\text { N }\end{array}$ & $\begin{array}{r}, 398^{\star \pi} \\
, 000 \\
75\end{array}$ & $\begin{array}{r}, 969^{\times \pi} \\
, 000 \\
75\end{array}$ & 1 \\
\hline
\end{tabular}

${ }^{* *}$ La correación es significativa en el nivel 0,01 ( 2 colas).

En el análisis correlacional entre los promedios de la calidad de vida según la felicidad y las dos escalas de satisfacción laboral se puede evidenciar que son positivas considerables, con valores de 0,886 para la primera escala y 0,884 para la segunda escala (tabla 3). Por tanto, la satisfacción laboral tiene una incidencia positiva con respecto a la calidad de vida desde la perspectiva de la felicidad.

\section{Tabla 3.}

Correlación calidad de vida según la felicidad - satisfacción laboral

\begin{tabular}{|c|c|c|c|c|}
\hline & & $\begin{array}{l}\text { Calidad de } \\
\text { vida - } \\
\text { felicidad }\end{array}$ & $\begin{array}{c}\text { Satisfacción } \\
\text { escala } 1\end{array}$ & $\begin{array}{l}\text { Satisfacción } \\
\text { escala } 2\end{array}$ \\
\hline \multirow[t]{3}{*}{ Calidad de vida - felicidad } & Correlación de Pearson & 1 & $886^{n \pi}$ &, $884^{\mathrm{n}}$ \\
\hline & Sig. (bilateral) & &, 000 &, 000 \\
\hline & $\mathrm{N}$ & 75 & 75 & 75 \\
\hline \multirow[t]{3}{*}{ Satisfacción escala 1} & Correlación de Pearson & $886^{k \pi}$ & 1 & $969^{k N}$ \\
\hline & Sig. (bilateral) &, 000 & &, 000 \\
\hline & $\mathrm{N}$ & 75 & 75 & 75 \\
\hline \multirow[t]{3}{*}{ Satisfacción escala 2} & Correlación de Pearson & $884^{\prime \prime}$ & $969^{\prime \prime}$ & 1 \\
\hline & Sig. (bilateral) &, 000 &, 000 & \\
\hline & $\mathrm{N}$ & 75 & 75 & 75 \\
\hline
\end{tabular}

${ }^{* *}$ La correación es significativa en el nivel 0,01 ( 2 colas).

En el análisis correlacional de la calidad de vida medida según la satisfacción con la vida, se pudo determinar que tiene una correlación positiva considerable con las dos escalas de satisfacción laboral, mostrando un valor de 0,762 para la primera y de 0,825 (tabla 4) para la 
segunda. Esto significa que las

personas que tienen una mejor satisfacción laboral tienen una mayor satisfacción con la vida.

Tabla 4.

Correlación calidad de vida según la satisfacción con la vida - satisfacción laboral

\begin{tabular}{|c|c|c|c|c|}
\hline & & $\begin{array}{c}\text { Satisfacción } \\
\text { de la vida }\end{array}$ & $\begin{array}{c}\text { Satisfacción } \\
\text { escala } 1\end{array}$ & $\begin{array}{c}\text { Satisfacción } \\
\text { escala } 2\end{array}$ \\
\hline \multirow[t]{3}{*}{ Satisfacción de la vida } & Correlación de Pearson & 1 &, $762^{n \pi}$ & $825^{n \pi}$ \\
\hline & Sig. (bilateral) & & 000 &, 000 \\
\hline & $N$ & 75 & 75 & 75 \\
\hline \multirow[t]{3}{*}{ Satisfacción escala 1} & Correlación de Pearson &, $762^{x \pi}$ & 1 &, $969^{n \pi}$ \\
\hline & Sig. (bilateral) &, 000 & &, 000 \\
\hline & $\mathrm{N}$ & 75 & 75 & 75 \\
\hline \multirow[t]{3}{*}{ Satisfacción escala 2} & Correlación de Pearson & $825^{2 \pi}$ & $969^{2 \pi}$ & 1 \\
\hline & Sig. (bilateral) &, 000 &, 000 & \\
\hline & $\mathrm{N}$ & 75 & 75 & 75 \\
\hline
\end{tabular}

${ }^{* *}$ La correación es significativa en el nivel 0,01 (2 colas).

En el análisis correlacional realizado entre la calidad de vida según la satisfacción de la situación económica y la satisfacción laboral, se logró identificar que esta dimensión se corelaciona de manera positiva media, obteniendo valores de 0,678 para la primera escala de satisfacción laboral y de 0,636 para la segunda escala (tabla 5). Estos valores reflejan que una mayor satisfacción laboral provoca una mayor satisfacción con la situación económica.

\section{Tabla 5.}

Correlación calidad de vida según la satisfacción de la situación económica satisfacción laboral

\begin{tabular}{|c|c|c|c|c|}
\hline & & $\begin{array}{l}\text { 4. Respecto } \\
\text { de su actual } \\
\text { situación } \\
\text { económica, } \\
\text { usted en } \\
\text { general está. }\end{array}$ & $\begin{array}{l}\text { Satisfacción } \\
\text { laboral } \\
\text { escala } 1\end{array}$ & $\begin{array}{l}\text { Satisfacción } \\
\text { laboral } \\
\text { escala } 2\end{array}$ \\
\hline $\begin{array}{l}\text { 4. Respecto de su actual } \\
\text { situación económica, } \\
\text { usted en general está. }\end{array}$ & $\begin{array}{l}\text { Correlación de Pearson } \\
\text { Sig. (bilateral) } \\
\mathrm{N}\end{array}$ & $\begin{array}{r}1 \\
75\end{array}$ & $\begin{array}{r}.678^{\prime \prime} \\
, 000 \\
75\end{array}$ & $\begin{array}{r}.636^{21} \\
, 000 \\
75\end{array}$ \\
\hline $\begin{array}{l}\text { Satisfacción laboral } \\
\text { escala } 1\end{array}$ & $\begin{array}{l}\text { Correlación de Pearson } \\
\text { Sig. (bilateral) } \\
\mathrm{N}\end{array}$ & $\begin{array}{r}.678^{\mathrm{N1}} \\
, 000 \\
75 \\
\end{array}$ & $\begin{array}{r}1 \\
75 \\
\end{array}$ & $\begin{array}{r}969^{\prime \prime} \\
, 000 \\
75 \\
\end{array}$ \\
\hline $\begin{array}{l}\text { Satisfacción laboral } \\
\text { escala } 2\end{array}$ & $\begin{array}{l}\text { Correlación de Pearson } \\
\text { Sig. (bilateral) } \\
\mathrm{N}\end{array}$ & $\begin{array}{r}.636^{\pi \times} \\
, 000 \\
75\end{array}$ & $\begin{array}{r}969^{n \pi} \\
, 000 \\
75\end{array}$ & $\begin{array}{r}1 \\
75\end{array}$ \\
\hline
\end{tabular}

${ }^{* *}$ La correación es significativa en el nivel 0,01 (2 colas). 
Por otra parte, en el análisis correlacional desarrollado según los aspectos sociodemográficos se pudo establecer que las únicas variables que se correlacionaron con la satisfacción laboral fueron el estado civil y el número de integrantes del hogar. En el caso del estado civil quienes estuvieron solteros, separados, divorciados 0 viudos reflejaron una menor insatisfacción que los casados o unidos; en tanto que a menor número de integrantes del hogar existe una mayor satisfacción laboral, tal como se muestra en la tabla 6. Las causas de estas relaciones superan el alcance de esta investigación.

Tabla 6.

Correlaciones de aspectos sociodemográficos con satisfacción laboral

\begin{tabular}{ccc}
\hline Pregunta & Escala 1 sl & Escala 2 s1 \\
\hline Estado civil & -0.398 & -0.391 \\
Edad & 0.017 & 0.041 \\
Género & 0.193 & 0.181 \\
Integrantes del hogar & -0.424 & -0.408 \\
Niños & 0.077 & 0.119 \\
Hijos propios & 0.099 & 0.150 \\
Nietos & -0.113 & -0.048 \\
\hline
\end{tabular}

Nota: El término s1 se refiere a satisafacción laboral. Los datos fueron tomados del programa informático SPSS.

En el análisis correlacional entre la calidad de vida según la satisfacción con la familia y las escalas de satisfacción laboral, se obtuvieron correlaciones positivas medias, con valores de 0,664 para la primera escala y 0,611 para la segunda. Estos resultados evidencian que una mayor satisfacción laboral viene relacionada a una mayor calidad de vida desde la satisfacción con la vida familiar.
En el análisis correlacional realizado entre el bienestar económico subjetivo como dimensión de la calidad de vida y la satisfacción laboral, se detectó una correlación positiva media de 0,606 y 0,651 para la primera y segunda escala, respectivamente. Estos resultados demuestran que el bienestar económico subjetivo tiene incidencia sobre la satisfacción laboral de la persona. 
En cuanto a la satisfacción de la vida familiar como dimensión de la calidad de vida y la satisfacción laboral, se pudo evidenciar que las correlaciones tuvieron valores de 0,880 para la primera escala y 0,844 para la segunda, representadas como correlaciones positivas considerables. Estos resultados demuestran que a medida que aumenta la satisfacción laboral se refleja un aumento en la satisfacción con la familia o viceversa.

La calidad de vida desde la dimensión de la felicidad obtuvo una valoración de 4,66, superior a la mediana de 4, por lo que se considera que la mayoría del personal de farmacia se siente feliz. En cuanto a las correlaciones con las dos escalas de satisfacción laboral se obtuvieron los valores de 0,886 y 0,884 , los cuales presentan correlación positiva considerable; por lo que se puede afirmar que la felicidad y la satisfacción laboral están correlacionadas.

La calidad de vida en su dimensión de la satisfacción con la vida presentó un valor de 4,27, el cual es superior a la mediana de 3,50 ; esto demuestra que mayoritariamente los colaboradores están satisfechos con sus vidas. Las correlaciones identificadas tuvieron valores de 0,762 y 0,825 , reflejando correlaciones positivas considerables; por tanto, se puede afirmar que la calidad de vida según la satisfacción con la vida guarda correlación con la satisfacción laboral.

La calidad de vida en su dimensión de satisfacción económica presentó una media de 3,49 , siendo similar a la mediana de 3,50; por consiguiente, se demuestra que la satisfacción por aspectos monetarios se encuentra dividida entre los colaboradores. Las correlaciones presentadas en esta dimensión fueron 0,678 y 0,636, siendo estas correlaciones positivas medias; por tanto, se puede afirmar que la calidad de vida según la satisfacción económica tiene relación con la satisfacción laboral.

Cabe mencionar que es primordial destacar que los trabajadores de las instituciones de salud juegan un rol clave en el desarrollo del sistema sanitario, debido a que un buen clima organizacional es indispensable para lograr un elevado rendimiento individual y colectivo del grupo 
humano de trabajo (Juárez-Adauta, 2012).

Respecto a los aspectos sociodemográficos se identificó que el estado civil tuvo correlaciones de 0,398 y -0,391, siendo estas correlaciones negativas débiles, en tanto que para los integrantes del hogar se obtuvieron valores de 0,424 y -0,408 siendo también correlaciones negativas débiles; esto significa que el estado civil y el número de integrantes del hogar se relaciona con la satisfacción laboral.

La calidad de vida en su dimensión de satisfacción con la vida familiar tuvo una media de 4,46, siendo superior a la mediana de 3,50, lo que refleja que los colaboradores están satisfechos con respecto a su familia. Las correlaciones presentaron valores de 0,664 y 0,611, ambas son correlaciones positivas medias; por lo que se puede afirmar que la calidad de vida según la satisfacción familiar se correlaciona con la satisfacción laboral.

La calidad de vida según el bienestar económico subjetivo tuvo una valoración de 2,29, siendo superior a la media de 2 , esto quiere decir que existe una satisfacción parcial con respecto a esta dimensión. En cuanto a la correlación, esta presentó valores de 0,606 y 0,651, por ende, se deduce una correlación positiva media; lo que significa que el bienestar económico subjetivo tiene correlación con la satisfacción laboral.

En cuanto a la calidad de vida según la satisfacción con la vida familiar en la segunda escala tuvo una media de 4,83, siendo esta mayor a la mediana de 4, lo cual representa que que los colaboradores están satisfechos en este aspecto. Las correlaciones presentaron valores de 0,880 y 0,844 ; infiriendo que se trata de correlaciones positivas considerables; por tanto, se puede afirmar que la satisfacción laboral y la satisfacción por la vida familiar están relacionadas, de forma similar a lo reportado por Suescún-Carrero et al. (2016), debido a que en este estudio se determinó una correlación positiva entre el trabajo, seguridad en el trabajo y la satisfacción de los trabajadores. No obstante, los resultados de la presente investigación presentan diferencias significativas con el reporte de Cogollo-Milanés \& GómezBustamante (2010), debido 
principalmente a que los trabajadores señalan que las políticas de promoción y remuneración son un factor clave en la percepción del trabajador y por tanto, se evidencian altos niveles de desmotivación con efectos considerables en la calidad de vida.

\section{Conclusiones}

Se concluye que la salud tiene una incidencia directa en la calidad de vida de la persona, además, se identificó que las organizaciones que no brindan reconocimiento a sus empleados ocasionan una baja satisfacción laboral, la remuneración tuvo incidencia en la satisfacción laboral, así como las relaciones interpersonales con los jefes y los compañeros.

Finalmente, todas las dimensiones de calidad de vida tuvieron respuestas afirmativas con respecto a esta variable, demostrando que el personal tiene una calidad de vida positiva. En cuanto a las correlaciones, todas las dimensiones de calidad de vida estuvieron correlacionadas con la satisfacción laboral, lo que demuestra su amplia relación e incidencia.

\section{Bibliografía}

Cogollo-Milanés, Z., GómezBustamante, E. (2010). Condiciones laborales en enfermeras de Cartagena, Colombia. Avances en Enfermería, 28(1):31-38.

Diener, E., Emmons, A., Larsen, R., \& Griffin, S. (1985). The satisfaction with life scale. Journal of Personality Assessment, 49(1), 71-75. Obtenido de http://labs.psychology.illinois. edu/ ediener/Documents/Die ner-Emmons-LarsenGriffin_1985.pdf

Giarrizzo, V. (2010). Bienestar económico subjetivo: Más allá del crecimiento. Red de Revistas Científicas de América Latina y el Caribe, España y Portugal, 34(28), 934.

doi:http://www.redalyc.org/ht ml/1956/195617795002/

Gómez, M., \& Sabeh, E. (2018). Calidad de vida. Evolución del concepto y su influencia en la investigación y la práctica. Obtenido de http://inico.usal.es/publicacion es/pdf/calidad.pdf

Hennessy, C., Moriarty, D., Zack, M., Scherr, P., \& Brackbill, R. (1994). Measuring healthrelated quality of life for public health surveillance. Public Health Rep, 109(5), 665-672. Obtenido 
https://www.ncbi.nlm.nih.gov/ pubmed/7938388

Instituto de Hematología e Inmunología. (2018). Metodología de la investigación. Obtenido de http://instituciones.sld.cu/ihi/m etodologia-de-lainvestigacion/

Juárez-Adauta, S. (2012). Clima organizacional y satisfacción laboral. Revista médica del instituto Mexicano del Seguro Social, 50(3), 307-314.

Lyubomirsky, S., King, L., \& Diener, E. (2005). The benefits of frequent positive affect: does happiness lead to success? Psychological Bulletin, 131(6), 803-855.

Mendoza-Mestanza, G. (2019). Calidad de vida y satisfacción laboral de los funcionarios del área de farmacia del Hospital Teodoro Maldonado Carbo, Ecuador. Revista Científica Arbitrada en Investigaciones de la Salud "GESTAR", 2(3), 2-22.

Pérez, J., \& Fidalgo, M. (1999). NTP 394: Satisfacción laboral: escala general de satisfacción. Obtenido de http://www.insht.es/InshtWeb/ Contenidos/Documentacion/F ichasTecnicas/NTP/Ficheros/ 301a400/ntp_394.pdf

Polanco, C. (2014). El clima y la satisfacción laboral en los(as) docentes del Instituto Tecnológico de Administración de Empresas
(INTAE) de la ciudad de San Pedro, Cortés. Repositorio de la Universidad Pedagógica Nacional Francisco Morazán. Obtenido de http://www.cervantesvirtual.co $\mathrm{m} /$ descargaPdf/el-clima-y-lasatisfaccionlaboral-en-losasdocentes-del-institutotecnologico-deadministracion-deempresasintae-de-la-ciudad-de.

Spector, P. (1997). Job satisfaction: Application, assessment, causes, and consequences. Oakland: Sage Publications, INC.

Suescún-Carrero, S., Sarmiento, G., Álvarez, L., \& Lugo, M. (2016). Calidad de vida laboral en trabajadores de una Empresa Social del Estado de Tunja, Colombia. Rev Med Risaralda, 22(1), 14-17.

Universitat de Valéncia. (2018). Escala de satisfacción con la vida (SWLS). Obtenido de https://www.uv.es/uipd/cuesti onarios/accesolibre/ESV.pdf

Vera, P., \& Celis, K. (2011). Evaluación de la felicidad: Análisis psicométrico de la escala de felicidad subjetiva en población chilena. Terapia Psicológica, 29(1), 127-133. Obtenido de http://www.redalyc.org/pdf/78 5/78518428013.pdf

Warr, P. (1999). Wellbeing: The Foundations of Hedonic 
Psychology. New York:

Russell Sage Foundation.

Zabriskie, R., \& Ward, P. (2013).

Satisfaction with family life

scale, marriage \& family.

49(5), $\quad$ 446-463.

doi:10.1080/01494929.2013.

768321 